\title{
Suspended SU-8 structures for monolithic microfluidic channels
}

\author{
Y. Moser $\cdot$ R. Forti $\cdot$ S. Jiguet $\cdot$ T. Lehnert $\cdot$ \\ M. A. M. Gijs
}

Received: 17 March 2010/Accepted: 20 June 2010/Published online: 9 July 2010

(C) Springer-Verlag 2010

\begin{abstract}
SU-8 photoresist is commonly used in the field of microfabrication as structural material or for molding of microfluidic devices. One major limitation, however, is the difficulty to process partially freestanding SU-8 structures or monolithic closed cavities and channels on-chip. We propose here a simple method for the fabrication of suspended structures, in particular of monolithic SU-8 microchannels. The method is based on the processing of a SU-8 doublelayer. Appropriate modification of the optical properties of the upper layer allows for selective crosslinking in the layer sandwich. This process is suitable for versatile layouts comprising open and hollow SU-8 structures on the same chip.
\end{abstract}

Keywords SU-8 - Monolithic microchannel ·

Double-layer

\section{Introduction}

The negative epoxy-based photoresist SU-8 has a wide range of applications in the field of microfabrication. The possibility to process up to several $100 \mu \mathrm{m}$ thick layers with high aspect ratio, as well as its optical transparency and chemical inertness explain the popularity of this material (Abgrall et al. 2007). SU-8 masters are frequently used for molding

Y. Moser $(\bowtie) \cdot$ R. Forti · T. Lehnert · M. A. M. Gijs Laboratory of Microsystems, Ecole Polytechnique Fédérale de Lausanne, Lausanne (EPFL), EPFL STI IMT LMIS2, BM 3133 (Bâtiment BM), Station 17, 1015 Lausanne, Switzerland e-mail: yves.moser@epfl.ch

\section{S. Jiguet}

Gersteltec, Pully, Switzerland of polydimethylsiloxane (PDMS) microfluidic channels (Jo et al. 2000). Even if standard SU-8 processing is routinely implemented in most microfabrication laboratories, a particular challenge consists in fabricating SU-8 structures with free-standing or suspended parts (Abgrall et al. 2007). In this technical note, we propose a new and robust technique to fabricate monolithic suspended SU-8 structures. In particular, we demonstrate the feasibility of creating closed SU-8 microfluidic channels on-chip, side-by-side with open structures. This degree of flexibility in SU-8 processing could not be achieved with other techniques presented so far.

Different approaches have been proposed in literature to fabricate SU-8 structures that are dissociated from the substrate. A positive photoresist (AZ-1518) was used as sacrificial layer for the fabrication of a SU-8 rotor for piezoelectric motors (Dellmann et al. 1998). This method, however, may not be generally applicable, as the thickness of the sacrificial layer does not exceed a few micrometers. Furthermore, it has been observed that solvent diffusion at the interface alters the SU-8 properties (Liu et al. 2004). Bonding of two SU-8 layers was successfully implemented for the fabrication of SU-8 microchannels (Agirregabiria et al. 2005; Steigert et al. 2008). With this technique patterned SU-8 films on two distinct wafers are brought in close contact and bonded by thermal treatment. This approach also allows for the fabrication of multilayer structures. Lamination of a dry film photoresist (Riston ${ }^{\circledR}$ ) (Heuschkel et al. 1998) or uncrosslinked SU-8 layers (Abgrall et al. 2006) was also reported to cover microchannels, but applying pressure on the substrate may make this process prone to failure.

Free-standing SU-8 structures can be obtained by selective polymerization of a top layer without affecting the underlying unexposed layer. The latter layer has the function of a sacrificial layer and can be dissolved to 
release the suspended part. Selective exposure of SU-8 is possible using e-beam lithography (Mali et al. 2006), laser writing (Yu et al. 2004), or deep-UV lithography (Ceyssens and Puers 2006). These approaches offer good results, but require particularly sophisticated equipments. Another successful method was the deposition of a thin aluminum film in between two SU-8 layers to stop light propagation during exposure of the top SU-8 layer (Ceyssens and Puers 2006). Partial exposure of SU-8 was also achieved by using substrates coated with an antireflection layer (Chuang et al. 2003) or by adding a positive resist (SC1827) to the SU-8 to increase UV-absorption (Foulds and Parameswaran 2006). Fabrication of short embedded microchannel (up to $1.6 \mathrm{~mm}$ ) using two epoxy-based photoresist layers with different photo acid generator was also demonstrated (Kitsara et al. 2006).

In this article, we report on a new approach for the fabrication of long SU-8 monolithic suspended structures by using standard photolithography. Our method is based on the selective enhancement of the UV-absorption in the modified top layer of a SU-8 double-layer structure. This simple approach allows the fabrication of both long monolithic closed SU-8 microchannels and large suspended membranes.

\section{Experimental}

\subsection{Materials and methods}

Our fabrication process is based on a SU-8 double-layer structure. Figure 1 schematically shows the process flow. Two SU-8 layers featuring different UV-absorption spectra are superposed and processed. First, a standard SU-8 layer (GM 1070, Gersteltec, Switzerland) is spin-coated on a silicon wafer and processed (thickness $50 \mu \mathrm{m}, 2,000 \mathrm{rpm}$ for $30 \mathrm{~s}, 400 \mathrm{~mJ} / \mathrm{cm}^{2}$, mask 1). For microfluidic applications, this layer will define the height of the microchannel (Fig. 1a). Parameters for soft and post-exposure bake are indicated in Table 1. The layer is not developed at this stage. Subsequently, the second SU-8 layer (GM 1060, Gersteltec) is spin-coated on top of the first one $(20 \mu \mathrm{m}$, $1,000 \mathrm{rpm}$ for $30 \mathrm{~s}$ ), soft-baked and exposed (Fig. 1b, mask $2,44 \mathrm{~mJ} / \mathrm{cm}^{2}$ ).

The second SU-8 layer defines the thickness and shape of the suspended membrane in the final monolithic device. In order to fabricate the suspended structure, the optical properties of the second SU-8 layer have been modified. Exposure of the top layer must not affect underlying unexposed parts. We solved this issue by using a SU-8 film with enhanced UV-absorption, as is shown schematically in Fig. 1b. Enhanced UV-absorption is achieved by increasing the photoinitiator concentration of the standard
Standard SU-8

Exposed standard SU-8

High-UV-absorption SU-8

Exposed high-UV-absorption SU-8
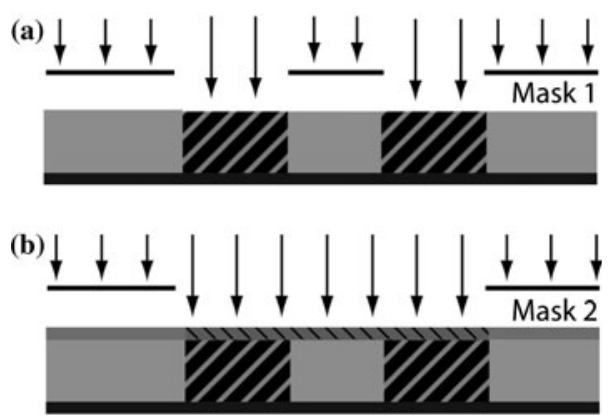

(c)

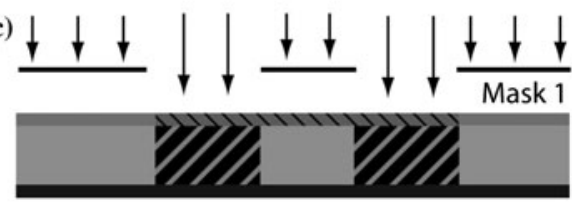

(d)

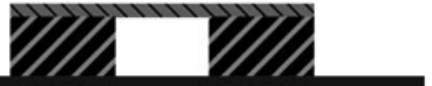

Fig. 1 Fabrication process of a suspended SU-8 structure for a monolithic closed SU-8 microchannel. a A standard SU-8 layer is spin-coated on a silicon wafer, followed by a soft-bake, exposure to define the channel walls (mask 1) and a post-bake. b A second layer using a modified high-UV-absorption SU-8 is spin-coated, soft-baked, and exposed to define the roof of the microchannel (mask 2). c Exposure through mask 1 is applied before the post-bake to improve the adhesion between the two SU-8 layers. $\mathbf{d}$ The SU-8 double layer is developed to release the microchannel

Table 1 Parameters for soft-bake (SB) and post-exposure bake (PEB) of the first SU-8 layer and the two superposed layers for the fabrication of suspended structures

\begin{tabular}{|c|c|c|c|}
\hline $\begin{array}{l}\text { 1st layer } \\
\text { Resist type: } \\
\text { GM } 1070\end{array}$ & & \multicolumn{2}{|c|}{$\begin{array}{l}1 \text { st }+2 \text { nd layer } \\
\text { Resist type: }\end{array}$} \\
\hline \multicolumn{4}{|l|}{ SB } \\
\hline $30 \Rightarrow 130^{\circ} \mathrm{C}$ & $50 \mathrm{~min}$ & $30 \Rightarrow 80^{\circ} \mathrm{C}$ & $25 \mathrm{~min}$ \\
\hline $130^{\circ} \mathrm{C}$ & $5 \mathrm{~min}$ & $80^{\circ} \mathrm{C}$ & $120 \mathrm{~min}$ \\
\hline $130 \Rightarrow 30^{\circ} \mathrm{C}$ & $50 \mathrm{~min}$ & $80 \Rightarrow 30^{\circ} \mathrm{C}$ & $25 \mathrm{~min}$ \\
\hline \multicolumn{4}{|l|}{ PEB } \\
\hline $30 \Rightarrow 80^{\circ} \mathrm{C}$ & $30 \mathrm{~min}$ & $30 \Rightarrow 90^{\circ} \mathrm{C}$ & $20 \min$ \\
\hline $80^{\circ} \mathrm{C}$ & $20 \min$ & $90^{\circ} \mathrm{C}$ & $50 \mathrm{~min}$ \\
\hline $80 \Rightarrow 30^{\circ} \mathrm{C}$ & $90 \mathrm{~min}$ & $90 \Rightarrow 30^{\circ} \mathrm{C}$ & $90 \mathrm{~min}$ \\
\hline
\end{tabular}

SU-8 (GM 1060) by a factor $\mathrm{k}(k=5$ or $k=10 ; k=1$ refers to the unmodified commercial resist). Different concentrations were prepared by mixing corresponding quantities of dissolved triarylsulfonium salt with SU-8. 
To ensure good adhesion between the two superposed patterned SU-8 layers in the final structure and full crosslinking of the top SU-8 layer is required at the interfaces. This is achieved by an additional exposure step through the first mask (Fig. 1c, mask 1, $30 \mathrm{~mJ} / \mathrm{cm}^{2}$ ). After a final postexposure bake, the wafer is developed in propylene glycol monomethyl ether acetate (PGMEA) for 16 min to release the microchannels and to remove encapsulated unpolymerized SU-8 (Fig. 1d). The developing time was experimentally determined in order to completely release the channel with the smallest cross-section $\left(50 \times 50 \mu \mathrm{m}^{2}\right)$. Soft-bake and post-exposure bake temperatures and ramps are adjusted experimentally and are summarized in Table 1. The soft-bake parameters of the second layer are most critical as they affect also the properties of the underlying first layer. In order to avoid overheating of the first layer, the maximum temperature of the post-exposure bake is reduced from $130^{\circ} \mathrm{C}$ (value recommended by the supplier) to $80^{\circ} \mathrm{C}$ and the time was increased from 5 to $120 \mathrm{~min}$

\subsection{Integrated monolithic microfluidic device}

Previously, we reported on an integrated microfluidic system for the retention and manipulation of magnetic particles in a microfluidic channel (Moser et al. 2009a, b). For that purpose, soft magnetic microtips had to be placed in close contact to the channel in order to generate high magnetic field strength and gradients. As schematically shown in Fig. 2, this device requires open recesses and sealed microfluidic structures in close contact on the same chip. The magnetic tips are typically $100 \mu \mathrm{m}$ wide and the channel sidewall thickness is $40 \mu \mathrm{m}$. This device is presented here as a representative example for a possible application of the new SU-8 process.

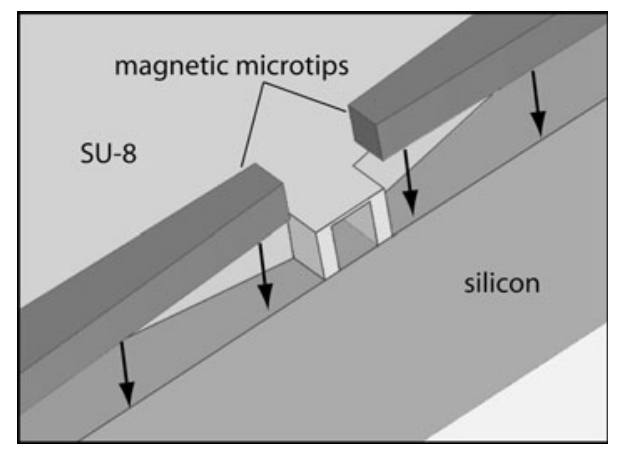

Fig. 2 Schematic view of a microfluidic device for magnetic particle handling on-chip. This device requires, on the same chip, a monolithic sealed SU-8 channel and open recesses for easy insertion from the top and accurate positioning of the magnetic microtips in close contact with the rigid channel sidewalls. A high-UV-absorption SU-8 layer is used to define the channel top membrane

\section{Results and discussion}

\subsection{Characterization of the modified SU-8 layers}

Increasing the photoinitiator concentration in the upper SU-8 layer enhances absorption of UV-light. We take advantage of this property to minimize crosslinking of the underlying SU-8 in the double-layer during exposure. Figure 3 shows measurements of the transmitted light through a $20 \mu \mathrm{m}$ thick SU-8 layer (GM 1060) on quartz in the range of $280-420 \mathrm{~nm}$. Transmission curves have been recorded for three different photoinitiator concentrations ( $k=1,5$ and 10) using a Lambda 900 spectrophotometer (Perkin Elmer, USA). For the wavelength of interest $(\mathrm{Hg}$ I-line, $365 \mathrm{~nm}$ ), the transmitted light for a standard SU-8 film $(k=1)$ is $87 \%$ of the incident light. A fivefold increase of the photoinitiator concentration $(k=5)$ results in a strong reduction of the transmitted light intensity to $69 \%$. Further increase of the photoinitiator concentration still reduces the transmitted intensity, but the effect is less pronounced (59\% for $k=10$ ).

It is important for the fabrication of suspended SU-8 structures to precisely control the thickness of polymerization when exposing the upper layer of the layer sandwich. The latter was determined by backside exposure of a $20 \mu \mathrm{m}$ thick single SU-8 film through a quartz wafer. Exposure at various doses results in polymerization of the film on the side in contact with the wafer, leaving an unpolymerized portion on top of it. After development, only the polymerized film remains on the wafer. Figure 4 shows the polymerization thickness as a function of exposure dose for two different photoinitiator concentrations (GM 1060, $k=1$ and $k=5$ ). Films with higher photoinitiator concentration $(k=10)$ show bad uniformity, most likely due to an excessive amount of solvent.

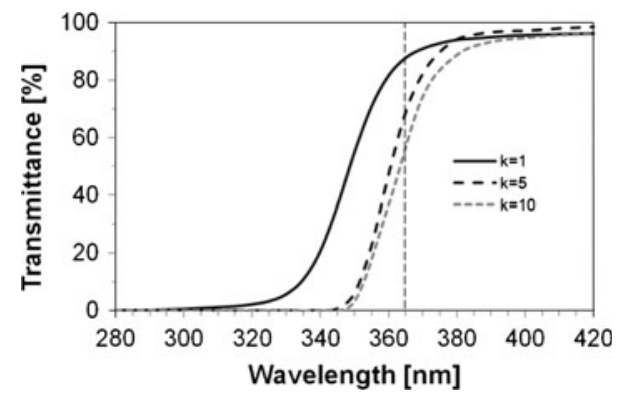

Fig. 3 Transmittance of $20 \mu \mathrm{m}$ thick SU-8 films (GM 1060) on quartz at different wavelengths as a function of the relative photoinitiator concentration $k$ ( $k=1$ refers to the standard concentration). In the modified films with 5 or 10 fold increase of the photoinitiator concentration a clear reduction of the transmittance from 87 to $59 \%$ is observed for the I line of the $\mathrm{Hg}$ spectrum $(\lambda=365 \mathrm{~nm})$ 


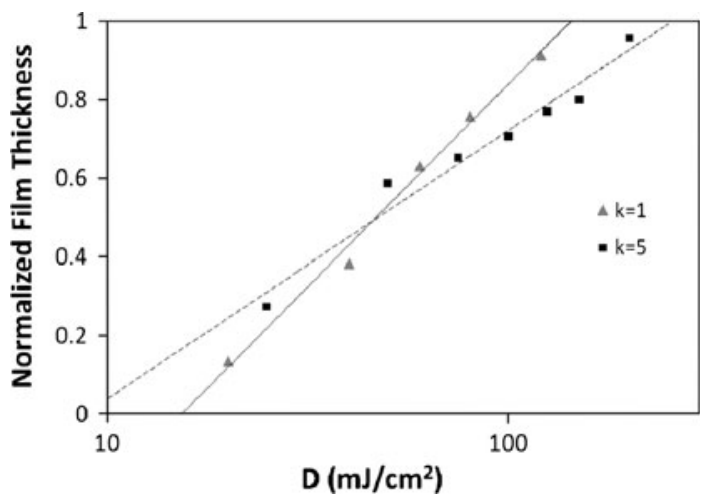

Fig. 4 Polymerized SU-8 film thickness as a function of the exposure dose $D$, as obtained by backside exposure after development $(\lambda=365 \mathrm{~nm}$, normalized with respect to the initial film thickness of $20 \mu \mathrm{m})$. Standard SU-8 ( $k=1$, GM 1060) and SU-8 with a 5 fold increased photoinitiator concentration $(k=5)$ are compared

The polymerization thickness as a function of the exposure dose is related to the contrast $\gamma$ of the resist,

$\gamma=\left[\log \frac{D^{0}}{D^{i}}\right]^{-1}$

where $D^{0}$ is the dose required to reach full polymerization of initial film thickness. The critical dose $D^{i}$ corresponds to the dose limit below which the complete SU-8 layer is dissolved during development (i.e., no polymerization occurred). In general, standard SU-8 formulations have high contrast, as is required for patterning thick layers with high aspect ratio. In our case, it is important to reduce the contrast of the top SU-8 layer in order to increase the window between the critical exposure dose $D^{i}$ and the exposure dose $D^{0}$ that leads to complete polymerization of the layer. The corresponding values can be derived from Fig. $4\left(D^{0}\right.$ for a normalized film thickness of 1 and $D^{i}$ for a normalized film thickness of 0 ). We find $D^{i}=15 \mathrm{~mJ} / \mathrm{cm}^{2}$ and $D^{0}=150 \mathrm{~mJ} / \mathrm{cm}^{2}$ for the standard SU-8 film $(k=1)$, and $D^{i}=9 \mathrm{~mJ} / \mathrm{cm}^{2}$ and $D^{0}=250 \mathrm{~mJ} / \mathrm{cm}^{2}$ for the modified SU-8 film (5 fold increased photoinitiator concentration, $k=5$ ), respectively. Using Eq. 1, we derive a contrast $\gamma$ of 0.7 for the modified film, compared to 1.0 for the standard film. Increasing the photoinitiator concentration thus decreases the slope of the dose-thickness curve in Fig. 4, allowing more precise control of the exposure of the modified top layer. As a consequence, residual polymerization of the underlying unexposed SU-8 can be efficiently avoided.

\subsection{SU-8 microchannels}

Double layer sandwiches comprising a standard SU-8 film $(k=1)$ and a modified SU-8 film $(k=5)$ are processed as shown in Fig. 1 to fabricate monolithic closed microfluidic channels on silicon substrates. The thickness of the lower layer, i.e., the channel height, is $50 \mu \mathrm{m}$ and the thickness of the top layer is $20 \mu \mathrm{m}$, respectively. The exposure dose of the modified film is adjusted experimentally to $44 \mathrm{~mJ} / \mathrm{cm}^{2}$. According to Fig. 4, this dose is sufficient to trigger crosslinking in about $10 \mu \mathrm{m}$ of the modified upper SU-8 layer. Crosslinking of underlying unexposed structures may therefore be securely avoided. These "sacrificial" parts are fully dissolved during development. The additional exposure step (Fig. 1c) allows fully polymerizing the $20 \mu \mathrm{m}$ top layer at areas that are attached to the underlying SU8 structures.

Figure 5 shows scanning electron microscope (SEM) pictures of the cross-section of three monolithic SU-8 microchannels that have been fabricated with the new method. The channel width is in the range of 50-200 $\mu \mathrm{m}$ with a length of $2 \mathrm{~cm}$. Pictures of the cross-sections are taken in the middle of the channel and show the complete
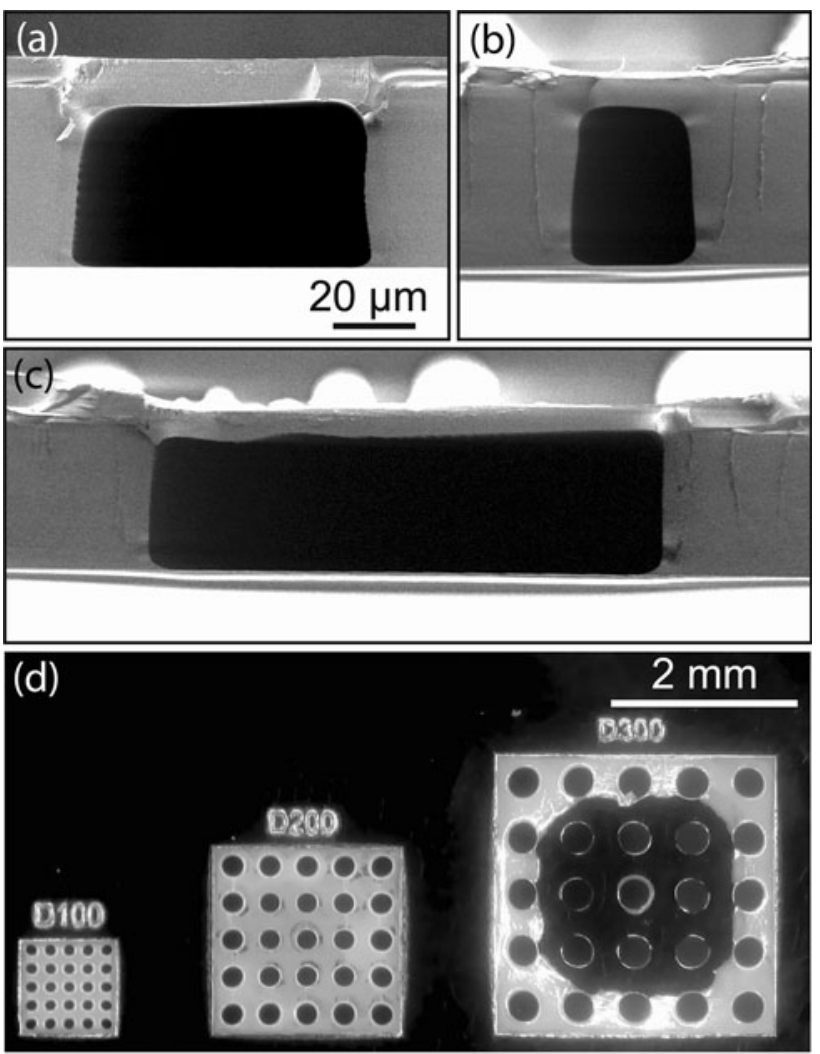

Fig. 5 a-c SEM pictures of cross-sections of different monolithic SU-8 channels fabricated using a SU-8 layer with modified optical properties for the suspended channel top membrane. The nominal cross-section areas are a $100 \times 50 \mu^{2}$, b $50 \times 50 \mu \mathrm{m}^{2}$, and c $200 \times 50 \mu \mathrm{m}^{2}$ with a mean channel roof thickness of $10 \mu \mathrm{m}$. The channel length is several centimeters. Pictures are taken in the middle of the channel. d Photograph of three membranes (thickness $10 \mu \mathrm{m}$ ) with a suspended area of $1 \times 1 \mathrm{~mm}^{2}, 2 \times 2 \mathrm{~mm}^{2}$ and $3 \times 3 \mathrm{~mm}^{2}$. The SU-8 membrane can withstand its own weight up to $2 \times 2 \mathrm{~mm}^{2}$, however, it collapsed for larger areas 



Fig. 6 Fluidic experiment showing the displacement of the meniscus of a water/air interface in a $100 \times 50 \mu \mathrm{m}^{2}$ channel at a flow rate of $0.5 \mathrm{nl} / \mathrm{s}$

dissolution and removal of encapsulated unpolymerized SU-8 in the channel. Due to the choice of the exposure dose, the resulting final thickness of the suspended structure, i.e., the roof of the microchannel, is about $10 \mu \mathrm{m}$. The required fine adjustment of the polymerized layer thickness is only possible with the modified SU-8. Similar experiments using a SU-8 top layer with standard properties were not successful. Large perforated membranes with a thickness of $10 \mu \mathrm{m}$ and an area up to $2 \times 2 \mathrm{~mm}^{2}$ could also be fabricated successfully (Fig. 5d). This demonstrates the feasibility of fabricating different kinds of suspended structures with this new method.

A fluidic experiment was carried out to verify the stability of the microchannel during flow injection. Water was injected in a $100 \times 50 \mu \mathrm{m}^{2}$ channel at $0.5 \mu \mathrm{l} / \mathrm{s}$ during $5 \mathrm{~min}$. Using these flow conditions the pressure drop along the microchannel is $\Delta p=1.4 \times 10^{4} \mathrm{~Pa}$. No leakages or damage of the microchannels were observed. The adhesion between the two superposed SU-8 layers is therefore sufficiently strong to carry out standard microfluidic experiments. Figure 6 shows the displacement of the meniscus of a water/air interface in the same channel. In this case, a flow rate of $0.5 \mathrm{nl} / \mathrm{s}$ was applied by suction through the fluidic connection via a syringe pump.

As an example for a possible application, we described above a device, in which robust and precise positioning of magnetic microtips close to a microfluidic channel is critical (see Fig. 2). A first device was made of commonly used micromoulded PDMS channels bonded on a glass slide. In this case, the magnetic parts had to be inserted from the side of the chip in between the glass and the PDMS part (Moser et al. 2009a, b). This operation is not suitable for reproducible and automated positioning of the magnetic parts.
Furthermore, the distance of the tips from the magnetic particle suspension in the channel is a critical parameter. It is defined by the sidewall thickness of the microchannel. However, thin PDMS sidewalls may be easily deformed when inserting the magnetic tips, resulting in inaccurate positioning and, in the worst case, in leaking microchannels. Our new fabrication technique for monolithic SU-8 structures provides a reliable solution for these issues.

\section{Conclusion}

We have developed a new and simple method for the fabrication of monolithic SU-8 microchannels and suspended SU-8 membranes. Only standard photolithographic equipment is required, the process can therefore be easily implemented in any microfabrication lab facility. The process is based on a SU-8 double layer structure comprising a top layer with enhanced UV-absorption. In this way, selective polymerization of the free-standing parts of a microstructure can be achieved. The present approach allows for high density integration of open, free-standing and hollow SU-8 structures on the same chip. This versatile approach opens new options for the design of microfluidic circuits or microdevices incorporating membranes. Certain drawbacks of commonly used PDMS microfluidic devices, related to the elasticity of the material for instance, as well as certain packing and interfacing issues, may be circumvented with this new technique.

Acknowledgments This work has been carried out in the frame of the European Commission funded project DETECTHIV (\# 037118, Sensitive nanoparticle assay for the detection of HIV). Gersteltec Sarl provided the modified SU- 8 photoresists. We also want to thank the staff of the Center of MicroNanoTechnology for their precious help during this work.

\section{References}

Abgrall P, Lattes C, Conedera V, Dollat X, Colin S, Gue AM (2006) A novel fabrication method of flexible and monolithic 3D microfluidic structures using lamination of SU-8 films. J Micromech Microeng 16(1):113-121

Abgrall P, Conedera V, Camon H, Gue AM, Nguyen NT (2007) SU-8 as a structural material for labs-on-chips and microelectromechanical systems. Electrophoresis 28(24):4539-4551

Agirregabiria M, Blanco FJ, Berganzo J, Arroyo MT, Fullaondo A, Mayora K, Ruano-Lopez JM (2005) Fabrication of SU-8 multilayer microstructures based on successive CMOS compatible adhesive bonding and releasing steps. Lab on a Chip 5(5):545-552

Ceyssens F, Puers R (2006) Creating multi-layered structures with freestanding parts in SU-8. J Micromech Microeng 16(6): S19-S23

Chuang YJ, Tseng FG, Cheng JH, Lin WK (2003) A novel fabrication method of embedded micro-channels by using SU-8 thick-film photoresists. Sens Actuators A 103(1-2):64-69 
Dellmann L, Roth S, Beuret C, Racine GA, Lorenz H, Despont M, Renaud P, Vettiger P, De Rooij NF (1998) Fabrication process of high aspect ratio elastic and SU-8 structures for piezoelectric motor applications. Sens Actuators A 70(1-2):42-47

Foulds IG, Parameswaran M (2006) A planar self-sacrificial multilayer SU-8-based MEMS process utilizing a UV-blocking layer for the creation of freely moving parts. J Micromech Microeng 16(10):2109-2115

Heuschkel MO, Guerin L, Buisson B, Bertrand D, Renaud P (1998) Buried microchannels in photopolymer for delivering of solutions to neurons in a network. Sens Actuators B 48(1-3):356361

Jo BH, Van Lerberghe LM, Motsegood KM, Beebe DJ (2000) Threedimensional micro-channel fabrication in polydimethylsiloxane (PDMS) elastomer. J Microelectromech Syst 9(1):76-81

Kitsara M, Chatzichristidi M, Niakoula D, Goustouridis D, Beltsios K, Argitis P, Raptis I (2006) Layer-by-layer UV micromachining methodology of epoxy resist embedded microchannels. Microelectron Eng 83(4-9):1298-1301

Liu J, Cai B, Zhu J, Chen D, Li Y, Zhang J, Ding G, Zhao X, Yang C (2004) A novel device of passive and fixed alignment of optical fiber. Microsyst Technol 10(4):269-271
Mali P, Sarkar A, Lal R (2006) Facile fabrication of microfluidic systems using electron beam lithography. Lab on a Chip 6(2):310-315

Moser Y, Lehnert T, Gijs MAM (2009a) On-chip immuno-agglutination assay with analyte capture by dynamic manipulation of superparamagnetic beads. Lab on a Chip 9:3261-3267

Moser Y, Lehnert T, Gijs MAM (2009b) Quadrupolar magnetic actuation of superparamagnetic particles for enhanced microfluidic perfusion. Appl Phys Lett 94:022505

Steigert J, Brett O, Muller C, Strasser M, Wangler N, Reinecke H, Daub M, Zengerle R (2008) A versatile and flexible lowtemperature full-wafer bonding process of monolithic 3D microfluidic structures in SU-8. J Microelectromech Syst 18(095013):095013

Yu H, Balogun O, Li B, Murray TW, Zhang X (2004) Building embedded microchannels using a single layered SU-8, and determining Young's modulus using a laser acoustic. J Microelectromech Syst 14:1576-1584 